\title{
Suppression of Primary Splenocyte Proliferation by Artemisia capillaris and Its Components
}

\author{
Hye Eun Lee', Gabsik Yang', Jae Sue Choi ${ }^{2}$ and Joo Young Lee' \\ ${ }^{1}$ BK21 plus team, College of Pharmacy, The Catholic University of Korea, Bucheon, Korea \\ ${ }^{2}$ Department of Food and Life Science, Pukyong National University, Busan, Korea
}

\begin{abstract}
The host immune system is the first line of host defense, consisting mainly of innate and adaptive immunity. Immunity must be maintained, orchestrated, and harmonized, since overactivation of immune responses can lead to inflammation and autoimmune diseases, while immune deficiency can lead to infectious diseases. We investigated the regulation of innate and adaptive immune cell activation by Artemisia capillaris and its components (ursolic acid, hyperoside, scopoletin, and scopolin). Macrophage phagocytic activity was determined using fluorescently labeled Escherichia coli, as an indicator of innate immune activation. Concanavalin A (ConA)- and lipopolysaccharide (LPS)-induced splenocyte proliferation was analyzed as surrogate markers for cellular and humoral adaptive immunity, respectively. Neither A. capillaris water extract (WAC) nor ethanol extract (EAC) greatly inhibited macrophage phagocytic activity. In contrast, WAC suppressed ConA- and LPS-induced proliferation of primary mouse splenocytes in a dosedependent manner. Similarly, EAC inhibited ConA- and LPS-induced splenocyte proliferation. Oral administration of WAC in mice decreased ConA- and LPS-induced splenocyte proliferation, while that of EAC suppressed LPS-induced splenocyte proliferation. Repeated administration of WAC in mice inhibited ConA- and LPS-induced splenocyte proliferation. Ursolic acid, scopoletin, and scopolin reduced ConA- and LPS-induced primary mouse splenocyte proliferation, while hyperoside did not show such activity. These results indicate that $A$. capillaris and its components, ursolic acid, scopoletin, and scopolin, suppress ConA- and LPS-induced adaptive immune cell activation. The results suggest that $A$. capillaris is useful as a regulator of adaptive immunity for diseases involving excessive immune response activation.
\end{abstract}

Key words: Immunosuppression, Innate immunity, Adaptive immunity, Natural product, Immune therapy

\section{INTRODUCTION}

Innate immune responses are the first line of defense against antigen invasion and include phagocytic activity and

\section{Correspondence to: Joo Young Lee, College of Pharmacy, The Catholic University of Korea, Bucheon 14662, Korea \\ E-mail: joolee@catholic.ac.kr}

Abbreviations: ConA, Concanavalin A; LPS, lipopolysaccharide; WAC, Artemisia capillaris water extract; EAC, Artemisia capillaris water ethanol extract; PAMPs, pathogen-associated molecular patterns; PRRs, pattern recognition receptors; DMEM, Dulbecco's modified Eagle's medium; FBS, fetal bovine serum; MTT, methylthiazol tetrazolium.

This is an Open-Access article distributed under the terms of the Creative Commons Attribution Non-Commercial License (http:// creativecommons.org/licenses/by-nc/3.0) which permits unrestricted non-commercial use, distribution, and reproduction in any medium, provided the original work is properly cited. antigen presentation, which guide and determine the adaptive immune response to antigen challenge. Host-pathogen interactions are typically initiated through host recognition of conserved molecular structures, known as pathogenassociated molecular patterns (PAMPs), which are essential for the pathogen life cycle. However, these PAMPs are deficient or compartmentalized in the host cell and are detected by the host's germline-encoded pattern recognition receptors (PRRs), which are expressed on the surface of innate immune cells, such as dendritic cells, macrophages, and neutrophils (1). Effective PAMP detection facilitates pathogen eradication by rapidly inducing host immune responses through the activation of complex signaling pathways to induce cytokine and chemokine-mediated inflammatory responses (2,3). A characteristic of adaptive immunity is the induction of a target effector response in two steps using antigen-specific receptors in $\mathrm{T}$ and $\mathrm{B}$ cells. First, antigens are presented to and recognized by antigen-specific $\mathrm{T}$ or $\mathrm{B}$ 
cells, leading to cell priming, activation, and differentiation, which usually occur in specific lymphoid tissue environments. Secondly, the activated $\mathrm{T}$ cells leave the lymphatic tissue at the infected site and return to the disease site, or the effector reaction occurs due to antibody release from activated B cells (plasma cells) into the blood and tissue fluid (4). Overactivation of the immune system can lead to inflammation and autoimmune disease. On the contrary, when the immune system is suppressed, it becomes vulnerable to infection and tumor development. It is therefore important to maintain an immune balance.

Artemisia capillaris is an herbal plant well-known for its various beneficial properties. It has been widely used as a hepatoprotective, analgesic, and antipyretic agent (5). Many studies have shown that $A$. capillaris has various biological activities, such as hypoglycemic (6), hypolipidemic (7), antiinflammatory (8), and anti-carcinogenic (9) effects. However, its immune-modulatory properties have not been fully examined. Therefore, we investigated whether a water or ethanol extract of $A$. capillaris and its components had immunosuppressive effects by modulating innate and adaptive immune cell activation using in vitro and ex vivo models. Our results may provide important information regarding immune balance regulation when consuming natural plant sources.

\section{MATERIALS AND METHODS}

Preparation of A. capillaris Thunb extracts. Whole $A$. capillaris plants were purchased from a local retailer and authenticated by Prof. J. H. Lee (Dongguk University, Kyeongju, Korea). The voucher specimen (No. 20090920) was deposited at the laboratory of Prof. J. S. Choi, a coauthor of this study. Whole plants were dried and ground into powder. The dried powder (each $100.0 \mathrm{~g}$ ) was then separately refluxed with $70 \%(\mathrm{v} / \mathrm{v})$ aqueous ethanol and water for $3 \mathrm{hr}(2 \times 0.5 \mathrm{~L})$ at $95^{\circ} \mathrm{C}$. The total filtrate was then concentrated to dryness in vacuo at $40^{\circ} \mathrm{C}$ to yield a $70 \%$ ethanol extract (EAC, $18.0 \mathrm{~g}$, yield: $18.0 \%$ ) and a water extract (WAC, $22.0 \mathrm{~g}$, yield: $22.0 \%$ ), respectively, which were then used in the pharmacological study.

Animals and cell culture. Male Balb/c mice, 7 weeks old, were obtained from Orientbio Inc (Seongnam, Gyeonggi, Korea). Animals were housed in groups of 5 per cage under standard animal housing conditions $\left(23^{\circ} \mathrm{C}, 12 \mathrm{hr} /\right.$ $12 \mathrm{hr}$ light/dark cycle, light on at 08:00) in the animal facility at the Catholic University of Korea. Mice were allowed to acclimatize for at least 1 week before experiments. Cell culture procedures were performed as previously described (10). Briefly, primary splenocytes isolated from mice and a murine monocytic cell line (RAW264.7; ATCC TIB-71, American Type Culture Collection, Manassas, VA, USA) were cultured in Dulbecco's modified Eagle's medium (DMEM) supplemented with $10 \%$ fetal bovine serum (FBS), $100 \mathrm{U} / \mathrm{mL}$ penicillin, and $100 \mu \mathrm{g} / \mathrm{mL}$ streptomycin. Cells were maintained in a humidified atmosphere under $5 \% \mathrm{CO}_{2}$ at $37^{\circ} \mathrm{C}$.

Phagocytosis assays. This was performed as described previously (11). RAW264.7 cells were plated in 96-well plates at $1 \times 10^{5}$ cells/well and incubated overnight. To quantify phagocytosis, cells were incubated with fluorescently-labeled Escherichia coli (K-12) BioParticles (Life Technologies Inc., Grand Island, NY, USA). Engulfed particles were enumerated after $1 \mathrm{hr}$ by measuring fluorescence emission per well at $480 \mathrm{~nm}$.

Splenocyte proliferation assay. This was performed as described previously (11). Primary splenocytes were isolated from Balb/c mouse spleens. Cells were seeded in 96well plates at $1 \times 10^{4}$ cells/well in complete DMEM. After stimulation with concanavalin A (ConA, $5 \mu \mathrm{g} / \mathrm{mL})$ or lipopolysaccharide (LPS, $10 \mu \mathrm{g} / \mathrm{mL}$ ), methylthiazol tetrazolium (MTT) assays (Sigma-Aldrich, St. Louis, MO, USA) were performed. MTT formazan product was determined using a microplate reader at an absorbance of $560 \mathrm{~nm}$ (Molecular Devices, San Francisco, CA, USA).

Histological assessment. Spleens were isolated from mice and fixed in $10 \%$ buffered formalin solution, dehydrated in ethanol, embedded in paraffin wax, sectioned, and stained with hematoxylin and eosin.

Statistical analyses. Data are presented as mean \pm SEM for the indicated numbers. Data comparisons between groups were examined using a student's $t$-test (significant when $p<0.05$ ).

\section{RESULTS}

A. capillaris does not suppress macrophage phagocytic activity. To investigate the effects of $A$. capillaris on innate immunity, WAC and EAC were prepared and their effects on macrophage phagocytic activity were determined as a representative indicator of innate immune response. Phagocytic activity was determined by the uptake of fluorescently-labeled $E$. coli in a mouse monocytic cell line (RAW 264.7). WAC treatment resulted in a slight decrease in phagocytic activity, by $20 \%$ maximum (Fig. 1A). EAC treatment reduced phagocytic activity to a similar degree (Fig. 1B). These results indicate that $A$. capillaris extracts do not significantly affect macrophage activity.

A. capillaris suppresses ConA- and LPS-induced proliferation of primary mouse splenocytes. Next, we investigated the effect of $A$. capillaris on the activation of adaptive immune cells, such as T and B lymphocytes. ConA is known as a $\mathrm{T}$ lymphocyte division promoter whereas LPS is a $\mathrm{B}$ 
(A)

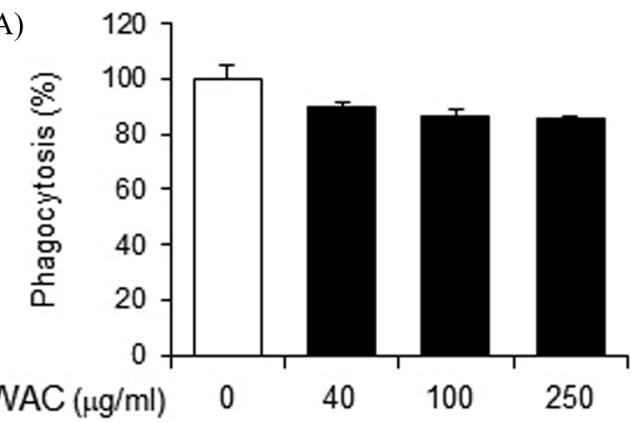

(B)

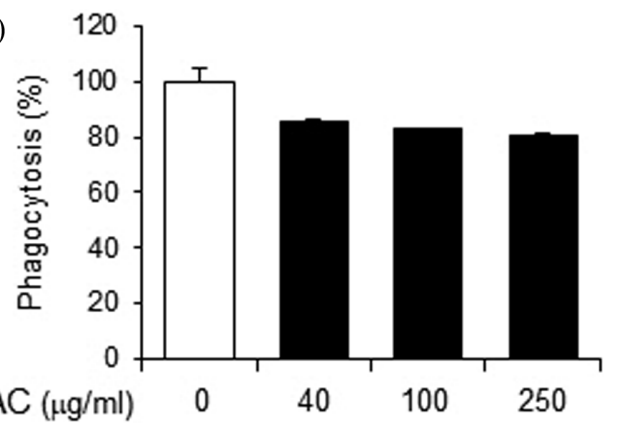

Fig. 1. Artemisia capillaris does not suppress phagocytic activity of macrophages. RAW264.7 cells were treated with (A) a water extract (WAC) or (B) an ethanol extract (EAC) of A. capillaris. Phagocytic activity was measured as the uptake of fluorescently-labeled Escherichia coli $(\mathrm{K}-12)$. Data are mean \pm SEM $(\mathrm{n}=3)$.

(A)

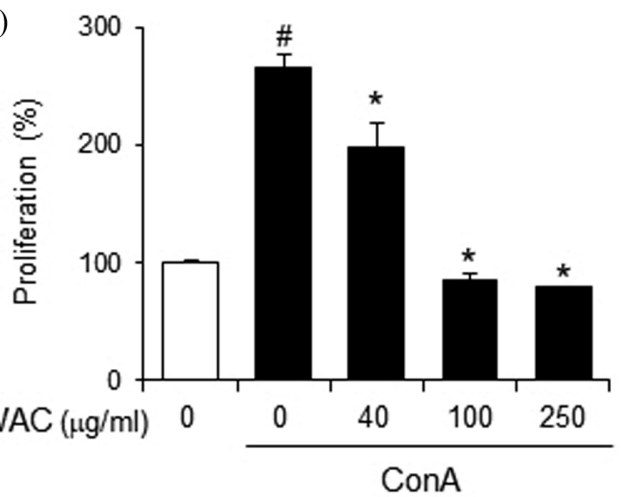

(C)

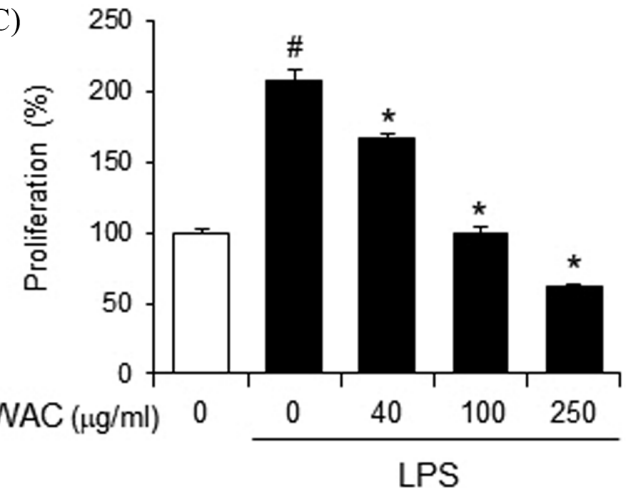

(B)

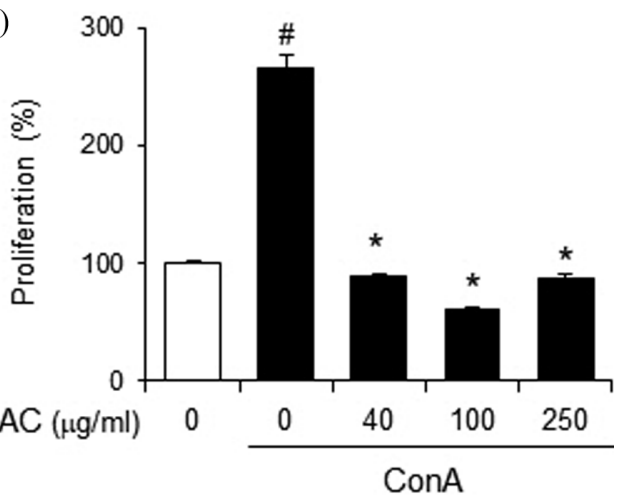

(D)

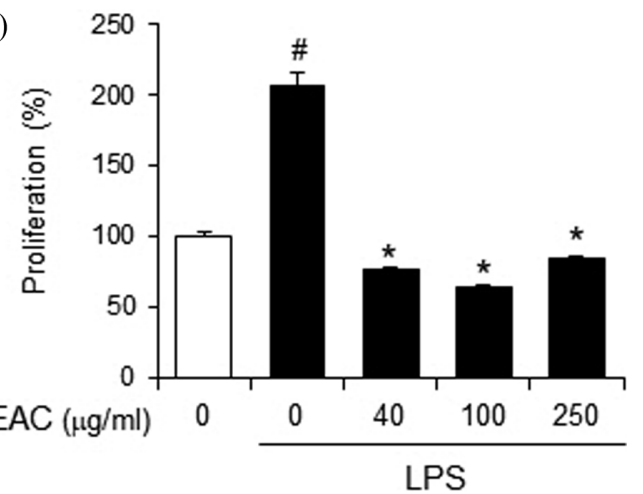

Fig. 2. Artemisia capillaris suppresses concanavalin A- and LPS-induced proliferation of primary mouse splenocytes. Primary splenocytes from Balb/c mice were stimulated with (A), (B). concanavalin A (ConA; $5 \mu \mathrm{g} / \mathrm{mL})$ or (C), (D). lipopolysaccharide (LPS; $10 \mu \mathrm{g} / \mathrm{mL})$ in the presence or absence of a (A), (C). water extract (WAC) or (B), (D). an ethanol extract (EAC) of A. capillaris for 24 hr. Cellular proliferation was measured by methylthiazol tetrazolium (MTT) assay. Data are mean \pm SEM $(n=3)$. "Significantly different from vehicle alone, $p<0.05$. *Significantly different from ConA or LPS alone, $p<0.05$. Veh, vehicle.

lymphocyte-activating factor. Therefore, ConA- and LPSinduced splenocyte proliferation was determined as parameters for cellular and humoral immunity, respectively. WAC decreased both ConA- and LPS-induced proliferation of primary mouse splenocytes in a dose-dependent manner (Fig. 2A and 2B). Similarly, EAC suppressed ConA- and LPSinduced splenocyte proliferation more potently than WAC (Fig. 2C and 2D). These results indicate that A. capillaris suppresses $\mathrm{T}$ and $\mathrm{B}$ lymphocyte activation, suggesting that A. capillaris treatment leads to a reduction in adaptive immune response, including cellular and humoral immune response.

Oral administration of A. capillaris extract suppresses ConA- and LPS-induced splenocyte proliferation. To confirm the inhibitory activity of $A$. capillaris on adaptive immunity, a single dose of $A$. capillaris extracts was orally 
administered to mice. Mouse splenocytes were isolated to examine whether A. capillaris intake affected ConA- or LPA-induced proliferation. WAC at 200 and $500 \mathrm{mg} / \mathrm{kg}$ reduced both ConA- and LPS-induced primary splenocyte proliferation, while WAC at $100 \mathrm{mg} / \mathrm{kg}$ did not inhibit sple- nocyte proliferation (Fig. 3A, 3B). Histological assessment of the mouse spleens did not show pathological changes in the WAC-treated groups (Fig. 3C). The spleen weight to body weight ratio slightly increased in the WAC-treated groups (Fig. 3D). However, no dose-response relationship

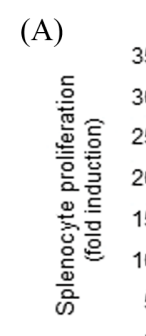

WAC $(\mathrm{mg} / \mathrm{kg})$

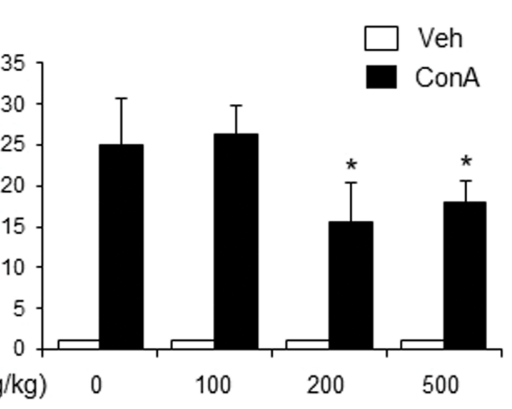

(C)

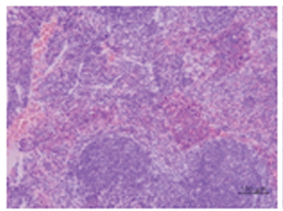

0

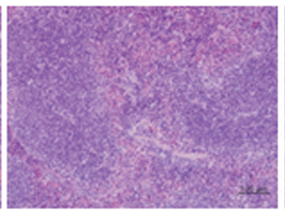

100

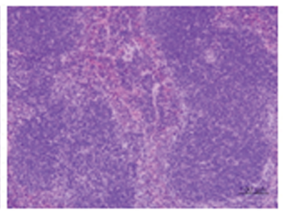

200

WAC (mg/kg)

(B)
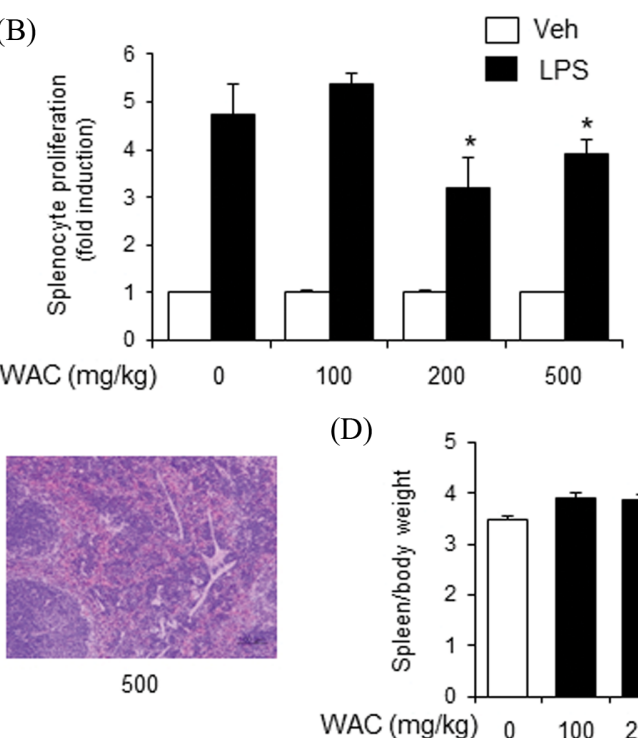

(D)

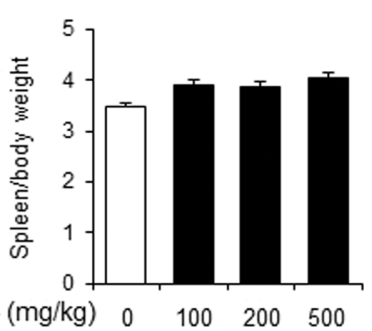

Fig. 3. Oral administration of Artemisia capillaris water extract reduces concanavalin A- and LPS-induced proliferation of primary mouse splenocytes. Balb/c mice were orally administered a water extract (WAC) of $A$. capillaris. (A), (B). After 24 hr, splenocytes were isolated and stimulated with concanavalin $A$ (ConA; $5 \mu \mathrm{g} / \mathrm{mL}$ ) or lipopolysaccharide (LPS; $10 \mu \mathrm{g} / \mathrm{mL}$ ). Cellular proliferation was measured by methylthiazol tetrazolium (MTT) assay. (C) Spleens were stained with hematoxylin and eosin. (D) Spleen weight per body weight. Data are mean \pm SEM $(n=3)$. *Significantly different from ConA or LPS alone, $p<0.05$. Veh, vehicle.

(A)

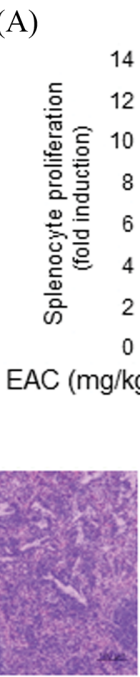

0

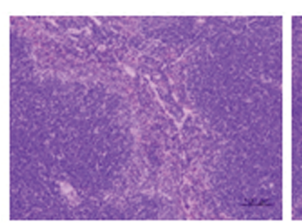

100

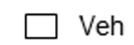

ConA

(C)

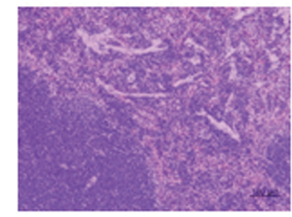

100

$\mathrm{EAC}(\mathrm{mg} / \mathrm{kg})$

(B)

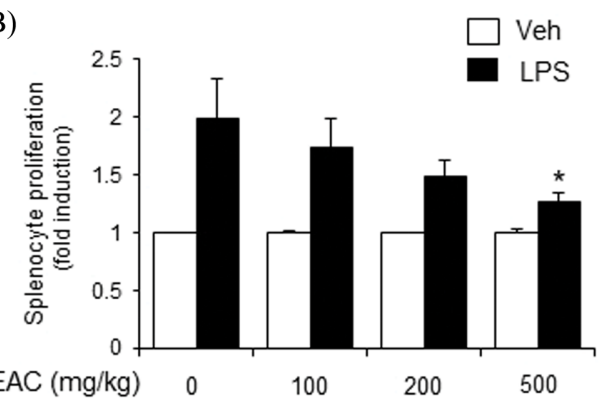

(D)

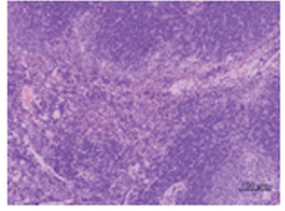

500

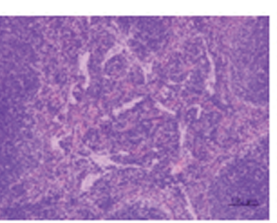

200

Fig. 4. Oral administration of Artemisia capillaris ethanol extract decreases LPS-induced primary mouse splenocyte proliferation. Balb/c mice were orally administered an ethanol extract (EAC) of A. capillaris. (A), (B). After $24 \mathrm{hr}$, splenocytes were isolated and stimulated with concanavalin A (ConA; $5 \mu \mathrm{g} / \mathrm{mL}$ ) or lipopolysaccharide (LPS; $10 \mu \mathrm{g} / \mathrm{mL})$. Cellular proliferation was measured by methylthiazol tetrazolium (MTT) assay. (C) Spleens were stained with hematoxylin and eosin. (D) Spleen weight per body weight. Data are mean \pm SEM $(n=3)$. *Significantly different from ConA or LPS alone, $p<0.05$. Veh, vehicle. 
(A)

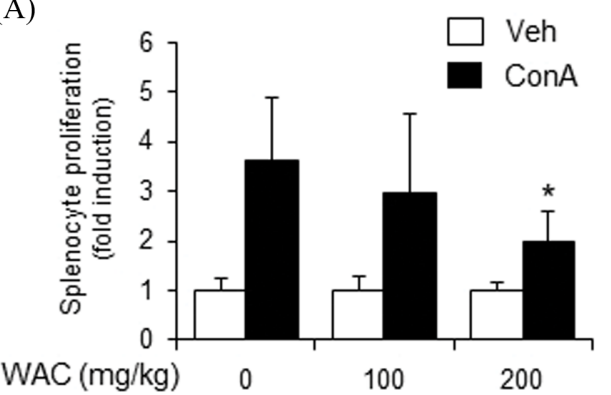

(C)

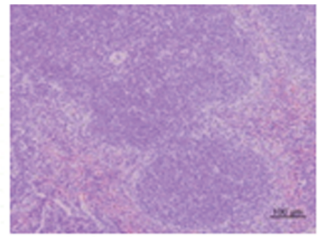

0

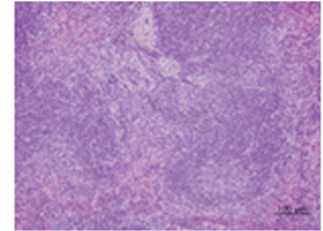

100
(B)

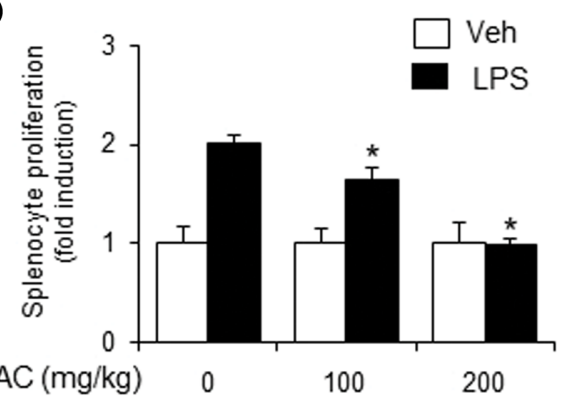

(D)

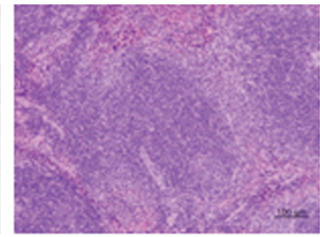

200

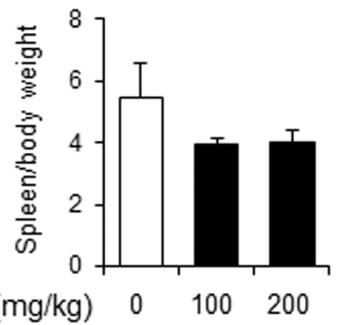

WAC $(\mathrm{mg} / \mathrm{kg})$

Fig. 5. Repeated administration of Artemisia capillaris water extract suppresses concanavalin A- and LPS-induced primary mouse splenocyte proliferation. Balb/c mice were orally administered a water extract (WAC) of A. capillaris once per day for 14 days. (A), (B). Splenocytes were isolated and stimulated with concanavalin A (ConA; $5 \mu \mathrm{g} / \mathrm{mL}$ ) or lipopolysaccharide (LPS; $10 \mu \mathrm{g} / \mathrm{mL}$ ). Cellular proliferation was measured by methylthiazol tetrazolium (MTT) assay. (C) Spleens were stained with hematoxylin and eosin. (D) Spleen weight per body weight. Data are mean \pm SEM $(n=3)$. *Significantly different from ConA or LPS alone, $p<0.05$. Veh, vehicle.

was observed (Fig. 3D).

EAC treatment did not inhibit ConA-induced primary splenocyte proliferation, but did reduce LPS-induced splenocyte proliferation (Fig. 4A, 4B). There were no significant histopathological changes in mouse spleens from the EAC-treated groups (Fig. 4C). The spleen weight to body weight ratio slightly decreased in the EAC-treated groups (Fig. 4D). However, there was no observable dose-response relationship (Fig. 4D).

We next investigated the effects of multiple A. capillaris exposures on adaptive immunity. Since inhibition was more potent with WAC treatment than with EAC treatment, WAC was orally administered to mice once per day for 14 days. Splenocytes were isolated and stimulated with ConA or LPS. ConA and LPS-induced proliferation was significantly attenuated by repeated administration of WAC (Fig. 5A, 5B). Histological evaluation of mouse spleen showed no noticeable changes in the treatment groups (Fig. 5C). The spleen weight to body weight ratio decreased in the EACtreated groups (Fig. 5D). However, this decrease was not dose-dependent (Fig. 5D).

These results indicate that orally administered $A$. capillaris results in the suppression of cellular and humoral adaptive immunity, without affecting spleen integrity.

Ursolic acid, scopoletin, and scopolin suppress ConAand LPS-induced splenocyte proliferation. We investigated whether A. capillaris components would affect sple- nocyte proliferation. Ursolic acid, scopoletin, and scopolin inhibited ConA-induced primary splenocyte proliferation, whereas hyperoside did not (Fig. 6A). Similarly, ursolic acid, scopoletin, and scopolin suppressed LPS-induced splenocyte proliferation, whereas hyperoside did not show a dosedependent inhibition (Fig. 6B). These results indicate that ursolic acid, scopoletin, and scopolin inhibit $\mathrm{T}$ and $\mathrm{B}$ lymphocyte activation, suggesting that these compounds inhibit cellular and humoral adaptive immune responses.

\section{DISCUSSION}

Immunosuppressive agents are a mainstay treatment for organ graft patients, and are becoming increasingly important in the treatment of autoimmune diseases (12). The use of immunosuppressive drugs is essential in cases of solid organ transplantation because it prevents an immune response against the graft, or delays the appearance of de novo baseline disease. The most frequently used drugs act on pathways that inhibit $\mathrm{T}$ cell proliferation and activation, the main mechanisms involved in rejection (13). Calcineurin inhibitors, such as cyclosporin A and tacrolimus, are the most commonly used treatments. Cyclosporin A emerged as an alternative to azathioprine, triggering an important advance in medical transplantation (14). These drugs inhibit the calcineurin pathway, preventing nuclear factor dephosphorylation in active $\mathrm{T}$ lymphocytes and subsequent transfer to the nucleus, ultimately blocking upregulation of genes 


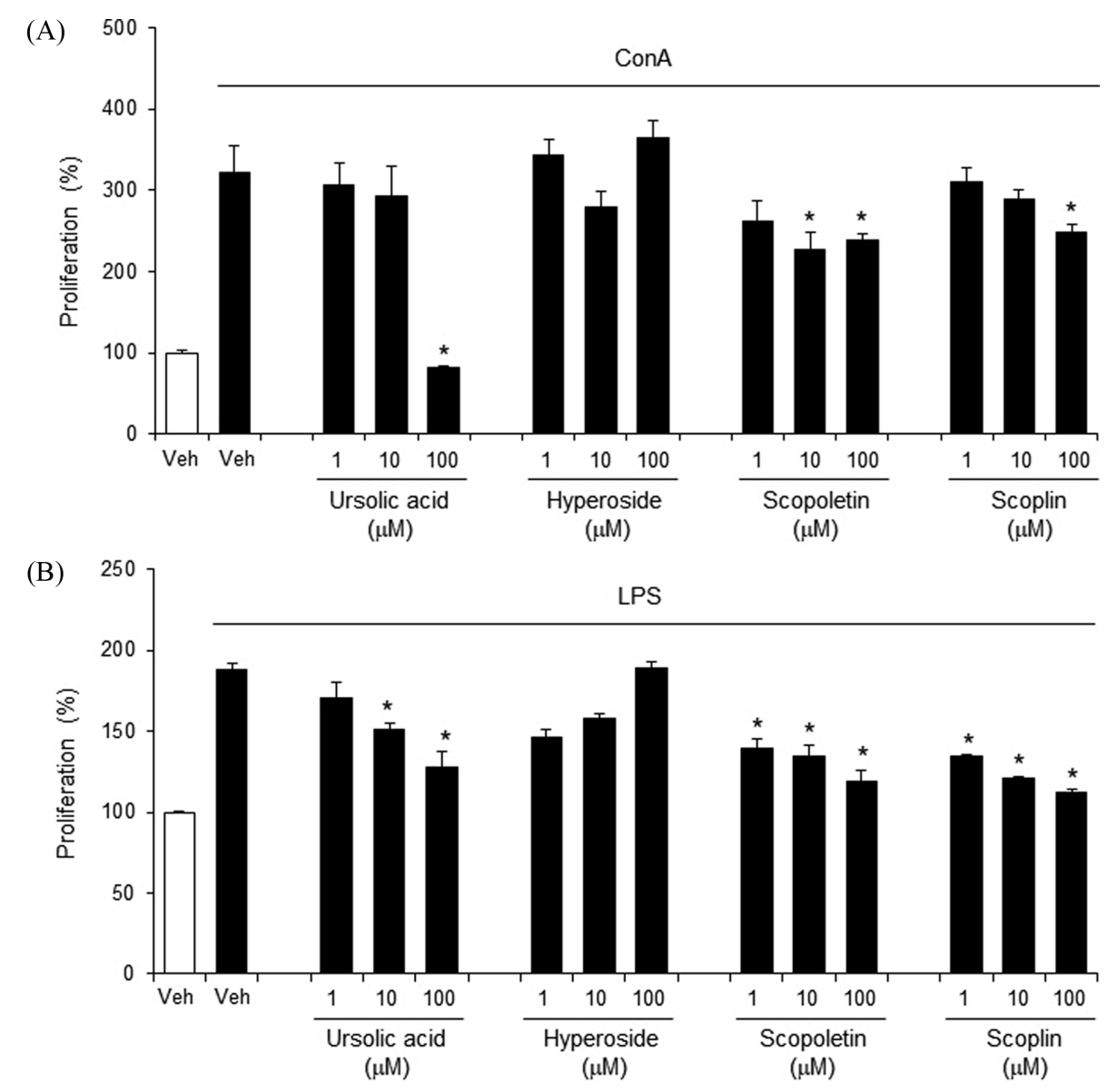

Fig. 6. Ursolic acid and scopoletin suppresses concanavalin A- and LPS-induced proliferation of primary mouse splenocytes. (A), (B). Primary splenocytes isolated from Balb/c mice were stimulated with concanavalin A (ConA; $5 \mu \mathrm{g} / \mathrm{mL}$ ) or lipopolysaccharide (LPS; $10 \mu \mathrm{g} /$ $\mathrm{mL}$ ) in the presence or absence of ursolic acid, hyperoside, scopoletin, and scopolin for $24 \mathrm{hr}$. Cellular proliferation was measured by methylthiazol tetrazolium (MTT) assay. Data are mean \pm SEM $(n=3)$. *Significantly different from ConA or LPS alone, $p<0.05$. Veh, vehicle.

involved in $\mathrm{T}$ cell activation, and consequently, immune response (15). Immunosuppressive drug therapy, although necessary after transplantation, has many adverse consequences, including side effects induced by secondary metabolite formation. Calcineurin inhibitors are associated with nephrotoxicity, cardiotoxicity and neurotoxicity; moreover, they increase the risk of many diseases after transplantation (16). Therefore, novel immune suppressants sourced from widely-used, traditional medicinal plants could be beneficial in providing clinically useful and safe treatments, with fewer side effects (17). Our results indicate that A. capillaris and its components (ursolic acid, scopoletin, scopolin) inhibit adaptive immune cell activation. These results suggest the potential use of $A$. capillaris and its components as immunosuppressants for diseases involving excessive activation of the adaptive immune system.

The genus Artemisia (family Asteraceae) includes over 500 species, and is found in Europe, North America, and mainly in Asia (18). Among them, A. capillaris is known in Chinese medicine as "Yin Chen Hao" (medicinal term "Injin"), and is the most important herb in East Asia, especially in Korea and China (19). The active components identified in A. capillaris extracts are caffeic acid, chlorogenic acid, peroxides, isocuritol, isochlorogenic acid A, and scopolin. Among them, chlorogenic acid, an ester of caffeic acid and quinic acid, has been reported as a main component of $A$. capillaris extract (20). In addition, various compounds have been reported including coumarins (scoparone, scopolin), flavonoids (isorhamnetin, quercetin, isoquercitrin, hyperoside), chromones (capillarisin, 7-methylcapillarisin), phenylpropanoids (caffeic acid, chlorogenic acid, caffeoylquinic acids), lignans $((+)$-sesamin, pluviatide, honokiol). and essential oils ( $\beta$-pinene, $\beta$-caryophyllene, capillene) $(5,21$ 24). Among these different constituents, we evaluated four compounds, including ursolic acid, hyperoside, scopoletin and scopolin (25-28), as modulators of adaptive immunity. Jang et al. (29) used response surface methodology to optimize the $A$. capillaris extraction parameters (extraction temperature, extraction time, and ethanol concentration) for obtaining an extract with high anti-inflammatory activity at 
the cellular level. The ranges used for determining the optimal extraction conditions were extraction temperatures of $57 \sim 65^{\circ} \mathrm{C}$, ethanol concentrations of $45 \sim 57 \%$, and extraction times of 5.5 6.8 hr (29). Yun et al. (30) investigated the 13week subchronic toxicity and genotoxicity of the $A$. capillaris extract. In the 13-week toxicity study, using dosages of $25,74,222,667$, and $2,000 \mathrm{mg} / \mathrm{kg}$ body weight, oral $A$. capillaris extract administration in male and female rats did not result in any significant adverse effects on food/water consumption, body weight, mortality, hematology, serum biochemistry, organ weight, and histopathology. Accordingly, the no-observed-adverse-effect level in rats of both genders was established for the A. capillaris extract at $2,000 \mathrm{mg} / \mathrm{kg} / \mathrm{day}$, the highest dose level tested. Yun et al. (30) demonstrated that the A. capillaris extract is considered safe for human consumption.

Collectively, our results demonstrate that $A$. capillaris and its active components suppress adaptive immune cell activation in in vitro and ex vivo systems. These results suggest that $A$. capillaris and its active components may be useful as, or in the development of, novel immunosuppressive treatments.

\section{ACKNOWLEDGMENTS}

We thank Eunshil Jeong and Jae Hyun Jun for their technical assistance. This study was supported by a grant from Basic Science Research Program through the National Research Foundation of Korea (NRF), funded by the Ministry of Education (2017R1A6A3A11032822 to G. Y.).

\section{CONFLICT OF INTEREST}

The authors declare that they have no conflicts of interest.

Received July 13, 2017; Revised August 25, 2017; Accepted August 30, 2017

\section{REFERENCES}

1. Kumar, H., Kawai, T. and Akira, S. (2011) Pathogen recognition by the innate immune system. Int. Rev. Immunol., 30, 1634.

2. Kawai, T. and Akira, S. (2010) The role of pattern-recognition receptors in innate immunity: update on Toll-like receptors. Nat. Immunol., 11, 373-384.

3. Takeuchi, O. and Akira, S. (2010) Pattern recognition receptors and inflammation. Cell, 140, 805-820.

4. Parkin, J. and Cohen, B. (2001) An overview of the immune system. Lancet, 357, 1777-1789.

5. Cha, J.D., Jeong, M.R., Jeong, S.I., Moon, S.E., Kim, J.Y., Kil, B.S. and Song, Y.H. (2005) Chemical composition and antimicrobial activity of the essential oils of Artemisia scoparia and A. capillaris. Planta Med., 71, 186-190.

6. Twaij, H.A. and Al-Badr, A.A. (1988) Hypoglycemic activity of Artemisia herba alba. J. Ethnopharmacol., 24, 123-126.

7. Lee, J., Chae, K., Ha, J., Park, B.Y., Lee, H.S., Jeong, S., Kim, M.Y. and Yoon, M. (2008) Regulation of obesity and lipid disorders by herbal extracts from Morus alba, Melissa officinalis, and Artemisia capillaris in high-fat diet-induced obese mice. J. Ethnopharmacol., 115, 263-270.

8. Kim, E.K., Kwon, K.B., Han, M.J., Song, M.Y., Lee, J.H., Lv, N., Choi, K.B., Ryu, D.G., Kim, K.S., Park, J.W. and Park, B.H. (2007) Inhibitory effect of Artemisia capillaris extract on cytokine-induced nitric oxide formation and cytotoxicity of RINm5F cells. Int. J. Mol. Med., 19, 535-540.

9. Kim, Y.S., Bahn, K.N., Hah, C.K., Gang, H.I. and Ha, Y.L. (2008) Inhibition of 7,12-dimethylbenz[a]anthracene induced mouse skin carcinogenesis by Artemisia capillaris. J. Food Sci., 73, T16-T20.

10. Lee, J.Y., Lee, B.H. and Lee, J.Y. (2015) Gambogic acid disrupts toll-like Receptor4 activation by blocking lipopolysaccharides binding to myeloid differentiation factor 2. Toxicol. Res., 31, 11-16.

11. Yang, G., Seo, E.K., Lee, J.H. and Lee, J.Y. (2015) Suppression of splenic lymphocyte proliferation by Eucommia ulmoides and genipin. Chem. Biodivers., 12, 538-546.

12. Ader, J.L. and Rostaing, L. (1998) Cyclosporin nephrotoxicity: pathophysiology and comparison with FK-506. Curr. Opin. Nephrol. Hypertens., 7, 539-545.

13. Halloran, P.F. (2004) Immunosuppressive drugs for kidney transplantation. N. Engl. J. Med., 351, 2715-2729.

14. Williams, D. and Haragsim, L. (2006) Calcineurin nephrotoxicity. Adv. Chronic Kidney Dis., 13, 47-55.

15. Kuklina, E.M. and Shirshev, S.V. (2001) Role of transcription factor NFAT in the immune response. Biochemistry Mosc., 66, 467-475.

16. Mika, A. and Stepnowski, P. (2016) Current methods of the analysis of immunosuppressive agents in clinical materials: A review. J. Pharm. Biomed. Anal., 127, 207-231.

17. Cho, K.S., Lim, Y.-r., Lee, K., Lee, J., Lee, J.H. and Lee, I.-S. (2017) Terpenes from Forests and Human Health. Toxicol. Res., 33, 97-106.

18. Doh, E.J., Paek, S.-H., Lee, G., Lee, M.-Y. and Oh, S.-E. (2016) Application of partial internal transcribed spacer sequences for the discrimination of Artemisia capillaris from other Artemisia species. Evid. Based Complement. Alternat. Med., 2016, 7043436.

19. Lim, D.W., Kim, Y.T., Jang, Y.J., Kim, Y.E. and Han, D. (2013) Anti-obesity effect of Artemisia capillaris extracts in high-fat diet-induced obese rats. Molecules, 18, 9241-9252.

20. Ha, H., Lee, H., Seo, C.S., Lim, H.S., Lee, J.K., Lee, M.Y. and Shin, H. (2014) Artemisia capillaris inhibits atopic dermatitislike skin lesions in Dermatophagoides farinae-sensitized Nc/ Nga mice. BMC Complement. Altern. Med., 14, 100.

21. Park, K.M., Li, Y., Kim, B., Zhang, H., Hwangbo, K., Piao, D.G., Chi, M.J., Woo, M.H., Choi, J.S., Lee, J.H., Moon, D.C., Chang, H.W., Kim, J.R. and Son, J.K. (2012) High-performance liquid chromatographic analysis for quantitation of marker compounds of Artemisia capillaris Thunb. Arch. Pharm. Res., 35, 2153-2162.

22. Sheu, S.J., Chieh, C.L. and Weng, W.C. (2001) Capillary electrophoretic determination of the constituents of Artemisiae Capillaris Herba. J. Chromatogr. A, 911, 285-293. 
23. Wu, T.S., Tsang, Z.J., Wu, P.L., Lin, F.W., Li, C.Y., Teng, C.M. and Lee, K.H. (2001) New constituents and antiplatelet aggregation and anti-HIV principles of Artemisia capillaris. Bioorg. Med. Chem., 9, 77-83.

24. Hong, J.H., Lee, J.W., Park, J.H. and Lee, I.S. (2007) Antioxidative and cytoprotective effects of Artemisia capillaris fractions. Biofactors, 31, 43-53.

25. Jyoti, M.A., Nam, K.W., Jang, W.S., Kim, Y.H., Kim, S.K., Lee, B.E. and Song, H.Y. (2016) Antimycobacterial activity of methanolic plant extract of Artemisia capillaris containing ursolic acid and hydroquinone against Mycobacterium tuberculosis. J. Infect. Chemother, 22, 200-208.

26. Lee, S.H., Lee, J.Y., Kwon, Y.I. and Jang, H.D. (2017) Antiosteoclastic activity of Artemisia capillaris thunb. extract depends upon attenuation of osteoclast differentiation and bone resorption-associated acidification due to chlorogenic acid, hyperoside, and scoparone. Int. J. Mol. Sci., 18, 322.

27. Choi, B.R., Kumar, S.K., Zhao, C., Zhang, L.T., Kim, C.Y., Lee, S.W., Jeon, J.H., Soní, K.K., Kim, S.H., Park, N.C., Kim,
H.K. and Park, J.K. (2015) Additive effects of Artemisia capillaris extract and scopoletin on the relaxation of penile corpus cavernosum smooth muscle. Int. J. Impot. Res., 2015. 27, 225-232.

28. Ali, M.Y., Jannat, S., Jung, H.A., Choi, R.J., Roy, A. and Choi, J.S. (2016) Anti-Alzheimer's disease potential of coumarins from Angelica decursiva and Artemisia capillaris and structure-activity analysis. Asian Pac. J. Trop. Med., 9, 103111.

29. Jang, M., Jeong, S.W., Kim, B.K. and Kim, J.C. (2015) Extraction optimization for obtaining Artemisia capillaris extract with high anti-inflammatory activity in RAW 264.7 macrophage cells. Biomed. Res. Int., 2015, 872718.

30. Yun, J.W., Kim, S.H., Kim, Y.S., You, J.R., Cho, E.Y., Yoon, J.H., Kwon, E., Ahn, J.H., Jang, J.J., Che, J.H. and Kang, B.C. (2017) A comprehensive study on in vitro and in vivo toxicological evaluation of Artemisia capillaris. Regul. Toxicol. Pharmacol., 88, 87-95. 